FETAL AND NEONATAL EDITION

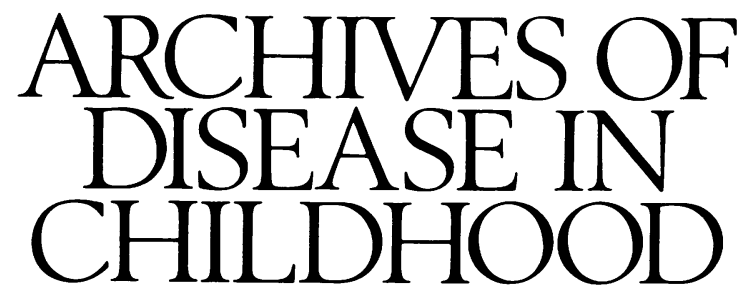

The fournal of the British Paediatric Association

\title{
Surgical management of neonatal necrotising enterocolitis
}

Neonatal necrotising enterocolitis (NEC) affects $1-2 \%$ of admissions to most neonatal units and is the commonest surgical emergency in the newborn. Premature infants with additional medical problems account for most cases but about $10 \%$ are term infants with a recognised risk factor such as congenital heart disease. ${ }^{12}$ NEC usually responds to intensive medical treatment, which includes cessation of feeding, gastrointestinal decompression, correction of hypovolaemia, coagulopathy and acidosis, broad spectrum antibiotics, ventilatory support if required, and parenteral nutrition. Between 30 and $50 \%$ of patients develop complications requiring surgery. ${ }^{1-4}$ There is little consensus on either the indications for or the timing and type of surgery.

\section{Indications for surgery}

Gastrointestinal perforation is the commonest and most widely accepted reason for operation. There is also general agreement that infants who continue to deteriorate over 12-24 hours despite maximum medical support should undergo surgery. Deterioration may be manifest by progressive abdominal tenderness and erythema and/or persistent acidosis, oliguria, and cardiovascular instability.

An abdominal mass or an abscess secondary to a localised perforation associated with increasing intestinal obstruction or persistent sepsis requires surgery. A fixed dilated loop of intestine may be visible on serial abdominal radiographs.

Inflammatory strictures develop in $10-30 \%$ of patients usually within three weeks to three months of medical or surgical treatment of NEC. ${ }^{5}$ Strictures causing obstruction may be multiple but are most often found in the distal colon. Although there are reports of successful balloon dilatation, ${ }^{7}$ excisional surgery is usually required.

Surgery is occasionally necessary when the diagnosis is in doubt. A neonate with a tender distended abdomen, bilious vomiting, bloody stools, and intramural gas on the plain abdominal radiograph is most likely to have NEC but the differential diagnosis includes midgut volvulus, ${ }^{8}$ Hirschsprung's enterocolitis, ${ }^{9}$ or rarely intussusception. ${ }^{10}$ Two other indications for surgery have been suggested but are not widely accepted. Evidence of portal vein gas on the plain abdominal radiograph is associated with a worse prognosis. Some of these patients respond to medical treatment alone but most eventually require operation. ${ }^{11}$ Some surgeons believe that early surgery in these cases may limit the evolution of intestinal necrosis and improve survival. ${ }^{12-14}$ The other doubtful area concerns the role of abdominal paracentesis. A positive tap of $0.5 \mathrm{ml}$ or more of brown fluid with visible bacteria on Gram stain is considered by some experts to indicate the presence of gangrenous bowel and the need for surgery. ${ }^{3615}$ However, false negative results may occur and this approach has not been shown to improve survival. ${ }^{15}$

\section{Surgical treatment}

Before surgery, all infants with NEC should have their T cryptantigen status assessed to avoid potentially fatal haemolysis in response to blood or plasma transfusion. ${ }^{16}$ During the past 30 years numerous operative approaches to NEC have been described which broadly fall into four categories. Peritoneal lavage is an integral part of any laparotomy in such patients.

(1) RESECTION OF NECROTIC/PERFORATED INTESTINE AND EXTERIORISING THE ENDS AS A PROXIMAL STOMA AND DISTAL MUCOUS FISTULA 361417

This technique conforms to standard surgical principles and is known to be effective but has several disadvantages. Restoration of intestinal continuity requires a second sep-arate operation with its own morbidity and mortality. ${ }^{5} 618$ With small bowel stomas in particular there is often difficulty re-establishing adequate enteral feeding and, despite the use of sodium supplements and loperamide, there exists the additional danger of serious fluid and electrolyte imbalance. ${ }^{6}{ }^{18-20}$ Other stoma specific problems include stenosis, prolapse, and skin excoriation. ${ }^{51820}$ Stoma closure is normally recommended when the infant is thriving but metabolic or mechanical problems often demand earlier surgery. Radiological assessment of the distal bowel before stoma closure is mandatory. 
(2) RESECTION AND PRIMARY ANASTOMOSIS

The traditional view is that intraperitoneal anastomoses heal badly in the presence of peritonitis, bowel distension, or compromised blood supply, all of which exist in NEC. ${ }^{621}$ However, several centres have now published retrospective reviews of the results of primary anastomosis in selected infants with NEC. ${ }^{192-25}$ With one exception, these series of non-randomised but similar patients indicate that primary anastomosis is associated with a lower mortality, fewer postoperative complications, a more rapid return to full enteral feeding, and a shorter hospital stay. Anastomotic leaks and strictures are uncommon with an extramucosal monofilament suture technique. ${ }^{2426}$ Moreover, there is a suggestion that the incidence of later strictures is decreased in comparison to that in defunctioned bowel. ${ }^{56}$ An anastomosis in inflamed but viable bowel heals well. ${ }^{2024} \mathrm{~A}$ theoretical disadvantage of primary anastomosis is that a more extensive resection is undertaken in order to ensure viable bowel ends ${ }^{6}$ but this has not been borne out in practice. ${ }^{20}$ Available evidence suggests that primary anastomosis in selected patients, that is stable infants with localised disease and viable bowel ends, is the procedure of choice.

\section{(3) PROXIMAL JEJUNOSTOMY}

A proximal defunctioning stoma is particularly valuable in the presence of extensive small bowel disease without definite necrosis ${ }^{122}$ or when attempted resection of an inflammatory mass could lead to life threatening blood loss. These situations are both associated with a high mortality. ${ }^{6} 24$

\section{(4) PERITONEAL DRAINAGE UNDER LOCAL ANAESTHESIA}

This is a useful adjunct to resuscitation in critically unstable premature infants weighing less than $1000 \mathrm{~g}$ with intestinal perforation. Under local anaesthesia, a soft Penrose drain is inserted into the iliac fossa. ${ }^{4}$ Drainage decompresses the abdomen, improves ventilation, and releases contaminated material. The infant is thus stabilised in preparation for later surgery and in some cases recovery is so dramatic that no further treatment is necessary. ${ }^{1428}$ Laparotomy should not be delayed if a patient fails to improve within 24 hours. ${ }^{29}$ Ein et al reported 37 such babies with intestinal perforation treated by drainage; one third made a complete recovery without further operation, one third died, and one third required subsequent surgery. ${ }^{28}$

Between 5 and $20 \%$ of surgically treated infants with NEC have extensive bowel ischaemia/necrosis (NEC totalis), often associated with widespread pneumatosis and portal vein gas on the plain abdominal radiograph. ${ }^{6} 143031$ This severity of disease is usually fatal and strategies such as a second look laparotomy, ${ }^{14}$ insertion of multiple drains, 814 or a decompressive high jejunostomy ${ }^{27}$ are rarely successful.

\section{Outcome after surgery}

Early postoperative recovery is frequently complicated by sepsis from indwelling cannulas and intra-abdominal sources, concomitant medical problems such as respiratory disease or intraventricular haemorrhage, and metabolic disturbances from stoma losses or parenteral nutrition. ${ }^{1} 6143132$ Nevertheless, the mortality from NEC continues to decline and in recent reports more than $70 \%$ of surgically managed infants have survived. ${ }^{614} 31$ Poor prognostic factors include extensive disease, addi- tional congenital anomalies, persistent acidosis, and renal failure. ${ }^{313334}$ Gestational age per se is of little importance, although infants under $1000 \mathrm{~g}$ fare worse. ${ }^{62933}$

In the long term, gastrointestinal adaptation and overall growth after surgery are usually good, ${ }^{631}$ but the later potential consequences of intestinal resection must be monitored. ${ }^{35} 36$ About $10 \%$ of surgically treated infants are severely limited by their short gut. ${ }^{631}$ Some degree of handicap is common among survivors, in part related to the general problems of prematurity. In one series of 40 patients who had undergone surgery for NEC, only half the survivors enjoyed good health, with various neurological disabilities accounting for much of the morbidity in the others. ${ }^{31}$

MARK D STRINGER LEWIS SPITZ

Department of Paediatric Surgery,

Institute of Child Health,

30 Guilford Street,

London WC1 1EH

Robertson JFR, Azmy AF, Young DG. Surgery for necrotizing enterocolitis. Brf Surg 1987; 74: 387-9.

2 Beeby PJ, Jeffery $\mathrm{H}$. Risk factors for necrotising enterocolitis: the influence of gestational age. Arch Dis Child 1992; 67: 432-5.

3 Kosloske AM. Surgery of necrotizing enterocolitis. World f Surg 1985; 9: 277-84.

4 Cheu HW, Sukarochana K, Lloyd DA. Peritoneal drainage for necrotizing enterocolitis. F Pediatr Surg 1988; 23: 557-61.

5 Musemeche CA, Kosloske AM, Ricketts RR. Enterostomy in necrotising enterocolitis: an analysis of techniques and timing of closure. $\mathcal{F}$ Pediatr Surg 1987; 22: 479-83.

6 Ricketts RR, Jerles ML. Neonatal necrotizing enterocolitis: experience with 100 consecutive surgical patients. World ₹ Surg 1990; 14: 600-5.

7 Renfrew DL, Smith WL, Pringle KC. Peranal balloon dilatation of a postnecrotizing enterocolitis stricture of the sigmoid colon. Pediatr Radiol 1986; 16: 320-2.

8 Moore TC. The management of necrotizing enterocolitis by 'patch, drain and wait'. Pediatric Surgery International 1989; 4: 110-4.

9 Ratta BS, Kiely EM, Spitz L, Brereton RJ. Improvements in the management of total colonic aganglionosis. Pediatric Surgery International 1990; 5 $30-6$.

10 Smith VS, Giacoia GP. Intussusception associated with necrotizing enterocolitis. Clin Pediatr (Phila) 1984; 23: 43-5.

11 Kurkchubasche AG, Smith SD, Rowe MI. Portal venous air - an old sign and new operative indication for necrotizing enterocolitis. Proceedings of the B A P S XXXVIII Annual International Congress. Budapest, Hungary the $B A P S X X X$

12 Martin LW, Neblett WW. Early operation with intestinal diversion for necrotizing enterocolitis. $\mathcal{F}$ Pediatr Surg 1981; 16: 252-5.

3 Cikrit D, Mastandrea J, Grosfeld JL, West KW, Schreiner RL. Significance of portal vein air in necrotizing enterocolitis: analysis of 53 cases. 7 Pediat Surg 1985; 20: 425-30.

14 Grosfeld JL, Cheu H, Schlatter M, West KW, Rescorla FJ. Changing trends in necrotizing enterocolitis. Ann Surg 1991; 214: 300-7.

15 Ricketts RR. The role of paracentesis in the management of infants with necrotizing enterocolitis. Am Surg 1986; 52: 61-5.

16 Novak RW, Abbott AE, Klein RL. T-cryptantigen determination affects mortality in necrotizing enterocolitis. Surg Gynecol Obstet 1993; 176: mortality

17 O'Neill JA, Stahlman MT, Meng HC. Necrotizing enterocolitis in the newborn: operative indications. Ann Surg 1975; 182: 274-9.

18 Cogbill TH, Millikan JS. Reconstitution of intestinal continuity after resection for neonatal necrotizing enterocolitis. Surg Gynecol Obstet 1985; 160: $330-4$

19 Rothstein FC, Halpin TC, Kliegman RJ, Izant RJ Jr. Importance of early ileostomy closure to prevent chronic salt and water losses after necrotizing enterocolitis. Pediatrics 1982; 70: 249-53.

20 Griffiths DM, Forbes DA, Pemberton PJ, Penn IA. Primary anastomosis fo necrotising enterocolitis: a 12-year experience. I Pediatr Surg 1989; 24 515-8.

21 Wayne ER, Burrington JD, Hutter J. Neonatal necrotizing enterocolitis. Arch Surg 1975; 110: 476-80.

22 Kiesewetter WB, Taghizadeh F, Bower RJ. Necrotizing enterocolitis: is there a place for resection and primary anastomosis? I Pediatr Surg 1979; 14: $360-3$.

23 Harberg FJ, McGill CW, Saleem MM, Halbert R, Anastassiou P. Resection with primary anastomosis for necrotizing enterocolitis. I Pediatr Surg with primary an

24 Sparnon AL, Kiely EM. Resection and primary anastomosis for necrotising enterocolitis. Pediatric Surgery International 1987; 2: 101-4

25 Cooper A, Ross AJ, O'Neill JA, Schnaufer L. Resection with primary anastomosis for necrotizing enterocolitis: a contrasting view. $\mathcal{J}$ Pediatr Surg 1988; 23: 64-8.

26 Brain AJL, Kiely EM. Use of a single layer extramucosal suture for intestinal anastomosis in children. Br $\mathcal{F}$ Surg 1985; 72: 483-4.

27 Firor HV. Use of high jejunostomy in extensive NEC. $\mathcal{f}$ Pediatr Surg 1982 17: 771-2.

28 Ein SH, Shandling B, Wesson D, Filler RM. A 13-year experience with peritoneal drainage under local anesthesia for necrotizing enterocolitis perforation. $₹$ Pediatr Surg 1990; 25: 1034-7.

29 Janik JS, Ein SH. Peritoneal drainage under local anesthesia for necrotizing enterocolitis (NEC) perforation: a second look. $\mathcal{F}$ Pediatr Surg 1980; 15: 565-8.

30 Kosloske AM, Musemeche MA, Ball WS, Ablin DS, Bhattacharyya N Necrotizing enterocolitis: value of radiographic findings to predict outcome $A 7 R$ 1988; 151: $771-4$ 
31 Jackman S, Brereton RJ, Wright VM. Results of surgical treatment of neonatal necrotizing enterocolitis. Br F Surg 1990; 77: 146-8.

32 Smith SD, Tagge EP, Miller J, Cheu H, Sukarochana K, Rowe MI. The hidden mortality in surgically treated necrotizing enterocolitis: fungal hidden mortality in surgically treated

33 Cikrit D, Mastandrea J, West KW, Schreiner RL, Grosfeld JL. Necrotizing enterocolitis: factors affecting mortality in 101 surgical cases. Surgery 1984; 96: 648-55.
34 Dykes EH, Gilmour WH, Azmy AF. Prediction of outcome following necrotizing enterocolitis in a neonatal surgical unit. $f$ Pediatr Surg 1985; 20: 3-5.

35 Valman HB. Intelligence after malnutrition caused by neonatal resection of ileum. Lancet 1974; i: 425-7.

36 Compston JE, Creamer B. The consequences of small intestinal resection. $Q \mathscr{f}$ Med 1977; 46: 485-97.

\section{Doppler and fetal growth retardation}

Intrauterine growth retardation (IUGR) is multifactorial in origin but it is well recognised that disordered uteroplacental and fetoplacental haemodynamics may be a feature in some cases. Doppler ultrasound employs a beam of ultrasound which is reflected by moving blood cells to produce a characteristic flow velocity waveform in which the spectral frequency shift of the reflected waveform reflects the velocity of the blood cells in the insonnated vessel. This technique now allows the safe non-invasive assessment of human uterine, placental, and fetal haemodynamics in normal and complicated pregnancies.

\section{Uteroplacental circulation}

Normally the non-pregnant spiral arteries undergo trophoblast invasion in two phases with loss of the elastic and vasoactive muscle coats in the decidual portion initially (phase I) and then as far as the radial arteries (phase II). This leads to a marked increase in uteroplacental blood flow and subsequent protection from the effects of circulating pressor agents.

In pre-eclampsia there is failure of the second wave of invasion causing retention of the spiral artery muscle coat, reduced perfusion of the intervillus space, maternal hypertension, and persisting responsiveness to circulating pressors leading to subsequent acute necrotising atherosis and further vessel occlusion. ${ }^{1}$

Similar defective trophoblast invasion has been found in $50 \%$ of placental bed biopsies from normotensive pregnancies complicated by IUGR ${ }^{2}$ suggesting that these placental vascular abnormalities and uteroplacental underperfusion constitute a significant but not a universal cause of IUGR

Uteroplacental Doppler blood flow studies have shown significant reduction in the diastolic velocities from the uterine artery and its major branches in some compromised human pregnancies and are thought to be related to extent of failed trophoblast invasion, increased uteroplacental impedance, and uterine ischaemia. ${ }^{3}$ The shape of uteroplacental Doppler waveforms is different from that in the umbilical artery. The rate is much less, being equivalent to the maternal pulse rate, the systolic phase is usually more peaked and there is often a prognostically significant diastolic notch in abnormal waveforms (see figure).

Continuous wave uteroplacental screening studies are not reproducible and pulsed Doppler studies are expensive and time consuming such that a useful role in screening is unlikely. Bewley and her colleagues employed a dual technique with continuous wave Doppler initially followed by colour and pulsed wave Doppler for suspected abnormal waveforms. ${ }^{4}$ This allows exclusion of many normal cases quickly and inexpensively using continuous wave Doppler and reduces the number who require the more tedious and exacting pulsed wave studies which would not be practical on a larger scale. They found them to be useful as early as 20 weeks' gestation in identifying those mothers who will deliver a fetus small for gestational age (sensitivity $36-45 \%$, specificity $87-97 \%$ ) or develop preeclampsia and this compares well with other similar screening studies.

\section{Umbilical artery circulation}

Morrow et al performed successive placental embolisation on chronically catheterised pregnant ewes using $50 \mu \mathrm{m}$ glass microspheres that became lodged in and occluded the arterioles of the placental tertiary villi. ${ }^{5}$ They found that the diastolic component of the umbilical artery Doppler flow velocity waveform became reduced, absent, and then reversed as embolisation proceeded, a pattern similar to that found in association with progressive IUGR and pre-eclampsia in humans. There was no association with increasing viscosity, hypoxia, hypoxia and acidosis, angiotensin II infusion (a proximal vessel constrictor), maternal hypertension, or reduced placental blood flow. The authors concluded that increased distal impedance was the likely cause of these abnormal waveforms as they were present in the embolisation experiments that increase the distal vascular resistance but could not be induced by any of the other listed factors. ${ }^{6}$ The mechanism of these waveform changes is thought to be a reflection of the forward propagated wave by the distal impedance which then reduces, cancels, or reverses the diastolic component of the waveform.

Umbilical artery waveforms are also influenced by cardiac contractility, vessel wall characteristics, and blood viscosity. ${ }^{7}$ Carunculectomy experiments, however, which reduce placental mass and blood flow leading to IUGR again suggest that the distal site of the vascular resistance is still the most important factor.

McCowan et al showed an inverse linear relationship between umbilical artery waveform indices and tertiary

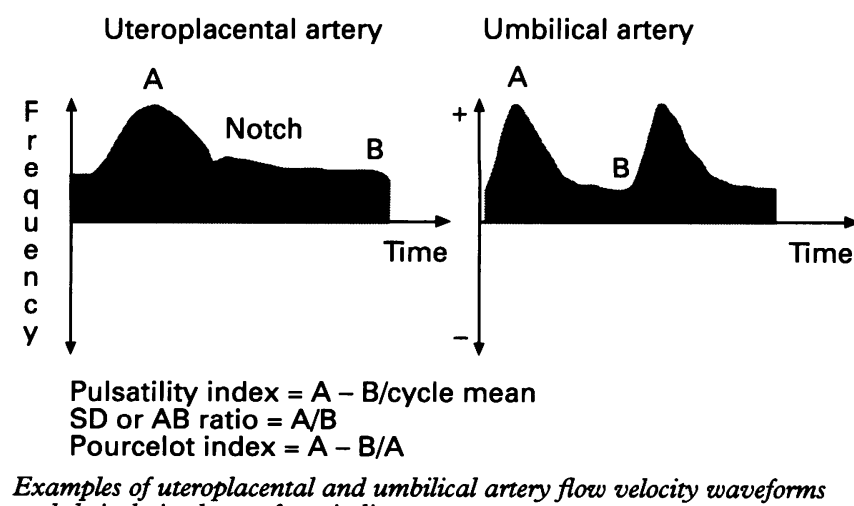
and their derived waveform indices. 